\title{
Exploring Margin Maximization for Biometric Score Fusion
}

\author{
Claudio Marrocco, Maria Teresa Ricamato, and Francesco Tortorella \\ DAEIMI - Università degli Studi di Cassino, Cassino, Italy \\ \{c.marrocco,mt.ricamato, tortorella\}@unicas.it
}

\begin{abstract}
Biometric systems are automated methods based on physical or behavioral characteristics of an individual for determining her/his identity. An important aspect of these systems is the reliability against forgery that is surely improved when using multiple sources of biometric information. In such cases combination rules can be applied to fuse the different scores thus obtaining a multibiometric system.

In this paper we analyze a method based on margin maximization for building a linear combination of biometric scores. The margin is a central concept in machine learning research and several theoretical results exist which show that improving the margin on the training set is beneficial for the generalization error of an ensemble of classifiers. Experiments performed on real biometric data and comparisons with other commonly employed fusion rules show that a combination based on margin maximization is particularly effective with respect to other established fusion methods.
\end{abstract}

Keywords: multiple classifiers systems, multibiometrics, margins, linear programming.

\section{Introduction}

Biometric systems aim to identify an individual by analyzing some physical characteristics such as fingerprints, iris, face, hand geometry, voice, etc. When dealing with a single source of biometric information, such systems could not ensure a sufficient degree of reliability because of the intrinsic noise affecting biometric data (e.g. a face can be partially occluded by sunglasses, fingerprints could vary due to different temperature and humidity conditions, etc.). Another problem is the vulnerability provided by only one characteristic which can be more easily counterfeited. An effective way to improve the accuracy is to combine multiple sources of biometric information: in this way more information is provided to the recognition/verification procedure and the whole process is more reliable and robust against spoofing attempts. Even though such combination could be performed at different levels, the most popular approach is to fuse the scores provided by a pool of classifiers (a.k.a.matchers) which are obtained by employing different feature extraction and/or matching techniques to different biometric characteristics. It is easy to see that such approach can be arranged in 
a Multiple Classifier System (MCS) framework, where the classification problem consists in discriminating between two categories: genuine and impostor. To this aim, several approaches have been proposed, based on nontrained combination rules [14, trained rules [1415], classifiers or density estimation [8].

Like all the other classification problems, even in multibiometrics a central problem is to guarantee a good generalization degree of the recognition/ verification system. In the area of the ensemble learning, the issue of generalization has been particularly considered in several papers aimed at explaining the reduction of the generalization error achieved by Adaboost. As it is well known, this algorithm generates weak classifiers via dinamically reweighting training samples on the basis of previous classification results; the final classifier is obtained by a convex combination of the constructed weak classifiers. In their analysis, Schapire et al. [12] introduced the concept of margin, which can be considered as a measure of the classification confidence, and showed that improving the margin on the training set guarantees an improvement in the upper bound on the generalization error of the ensemble of classifiers.

However these studies have been focused on Boosting algorithms which construct from scratch an ensemble of classifiers as different instances of a same base learning algorithm. The aim of our paper, instead, is to experimentally verify if the margin maximization can be beneficial also in biometric score fusion, where the classifiers in principle have different architectures, work on different sets of features and have been trained independently. To this aim, we have employed an algorithm for calculating the weights of a convex combination of matchers so as to maximize the margin of the combination on the training set. Several experiments performed on publicly available datasets have shown that this method is particularly effective if compared with other established fusion methods.

The rest of the paper is organized as follows: in sect. 2 the concept of margin and its characteristics are briefly recalled while in sect. 3 the method for maximizing the margin is described. In sect. 4 the results obtained on some publicly available data sets are presented and discussed. Sect. 5 concludes the paper with a summary and an outline of further research.

\section{Margins and Generalization}

Let us consider a two-class classification problem defined on an instance space $X$, in which the classes are identified by the two labels $\{-1,+1\}$. A classifier $f$ can be described as a mapping from $X$ to the interval $[-1,+1]$ such that a sample $\mathbf{x} \in X$ is assigned to one of the classes according to $\operatorname{sgn}(f(\mathbf{x}))$. If we assume that $y$ is the correct label of $\mathbf{x}$, the sample margin associated to $\mathbf{x}$ is given by $y f(\mathbf{x})$. As a consequence, $f$ provides a wrong prediction for $\mathbf{x}$ if the sample margin is negative. More precisely, while the sign of the margin indicates the correctness of the classifier, the magnitude estimates the confidence of the classifier in making its prediction on the sample $\mathbf{x}$. In this way, a large negative margin means that the classifier is wrong but highly confident. 
Let us now consider a training set $S$ containing $N$ samples $\mathbf{x}_{i}$ where $i=$ $1, \cdots, N$, each associated to a label $y_{i} \in\{-1,+1\}$. The minimum margin or classifier margin for $f$ over the set $S$ is defined as: $\mu(f)=\min _{i} y_{i} f\left(\mathbf{x}_{i}\right)$ The classifier margin has an intuitive meaning [4]: it measures the distance that the classifier can travel in the feature space without changing the way it labels any of the sample points. Moreover, it has a direct connection with the generalization capability of the classifier as proved in several papers 2313 which suggested upper bounds on generalization error expressed in terms of the classifier margin.

The margin theory has been also used in ensemble learning to analyze the generalization effectiveness of Adaboost. In particular, in 12 it has been proved that the error of a convex combination of classifiers is bounded by $\operatorname{Pr}_{S}[y f(\mathbf{x}) \leq \theta]$, i.e. the part of training examples with margin below a threshold $\theta$, plus a "complexity penalty" term which is $O(1 / \theta)$. In other words, the size of the minimum margin (i.e. the margin related to the worst result on the training set) appears to be the most relevant factor for improving generalization. On this basis, some experiments have been done aimed at improving the generalization performance of Adaboost by using a combination of the generated classifiers explicitly based on minimum margin maximization [5. However, the results showed that the modified version of Adaboost performs generally worse than the original one. A possible reason is that the boosting approach focuses on the most difficult samples and tries to improve their margins, but with a significant possibility of overfitting when dealing with very difficult samples [11. This problem could be further emphasized by a minimum margin maximization combination which would give higher weights to the classifiers with higher minimum margins, but more prone to overfitting.

However, as far as we know, the potential effectiveness of such a combination has not yet been examined when the classifiers of the ensemble are built independently and not according to a boosting approach and this becomes particularly interesting in the case of biometric score fusion.

\section{Margin Maximization Via Linear Programming}

In this section we describe the algorithm used for estimating the weights of a convex combination of classifiers which maximizes the minimum margin of the resulting classifier. As said in the previous section, the algorithm has been already used in [5] for experiments on Adaboost.

Let us consider again the training set $S$ containing $N$ samples $\left(\mathbf{x}_{i}, y_{i}\right)$. Let us moreover assume that $K$ already trained classifiers are available $f_{j}(\mathbf{x}) \rightarrow$ $[-1,+1]$ with $j=1, \cdots, K$. The margin provided by the $j$-th classifier over the $i$-th sample is defined as:

$$
\mu_{i j}=y_{i} \cdot f_{j}\left(\mathbf{x}_{i}\right)
$$

so that the $f_{j}$ correctly classifies $\mathbf{x}_{i}$ iff $\mu_{i j}>0$. Let us now consider the linear combination of the $K$ classifiers: 


$$
f_{c}(\mathbf{x})=\sum_{j=1}^{K} w_{j} f_{j}(\mathbf{x})
$$

with $w_{j} \geq 0$ and $\sum_{j=1}^{K} w_{j}=1$. The margin provided by $f_{c}$ over the $i$-th sample is thus

$$
\mu_{i}=y_{i} \cdot\left(\sum_{j=1}^{K} w_{j} f_{j}\left(\mathbf{x}_{i}\right)\right)=\sum_{j=1}^{K} w_{j} \mu_{i j}
$$

while the margin of $f_{c}$ is $\mu=\min _{i} \mu_{i}$. Actually the margin $\mu$ depends on the weights $\mathbf{w}=\left\{w_{1}, w_{2}, \cdots, w_{K}\right\}$ and thus such weights can be chosen to make the margin as large as possible. In this way we have a max-min problem which can be written as:

$$
\begin{aligned}
& \operatorname{maximize}\left(\min _{i} \sum_{j=1}^{K} w_{j} \mu_{i j}\right) \\
& \text { subject to } \quad \sum_{j=1}^{K} w_{j}=1 \\
& w_{j} \geq 0 \quad j=1,2, \cdots, K
\end{aligned}
$$

The problem can be recast as a linear problem [16] if we introduce the margin $\mu$ as a new variable:

maximize

$\mu$

subject to

$$
\begin{aligned}
& \sum_{j=1}^{K} w_{j} \mu_{i j} \geq \mu i=1,2, \cdots, N \\
& \sum_{j=1}^{K} w_{j}=1 \\
& w_{j} \geq 0 \quad j=1,2, \cdots, K
\end{aligned}
$$

If we collect the margins in a $N \times K$ matrix $\mathbf{M}=\left\{\mu_{i j}\right\}$, the weights in a vector $\mathbf{w}$ and define $\mathbf{e}_{t}$ the column vector consisting of $t$ ones and $\mathbf{z}_{t}$ the column vector consisting of $t$ zeros, the problem can be written in block-matrix form:

$$
\text { maximize } \quad\left[\begin{array}{ll}
\mathbf{z}_{K}^{T} & 1
\end{array}\right]\left[\begin{array}{l}
\mathbf{w} \\
\mu
\end{array}\right]
$$

subject to

$$
\begin{gathered}
{\left[\begin{array}{cc}
-\mathbf{M} & \mathbf{e}_{N} \\
\mathbf{e}_{N}^{T} & 0
\end{array}\right]\left[\begin{array}{l}
\mathbf{w} \\
\mu
\end{array}\right]} \\
\mathbf{w} \geq \mathbf{z}_{K}
\end{gathered}
$$

In principle, we could use any one of the numerous linear programming solving methods available, but it should be taken into account that the number of constraints could be very large since it equals the number of training samples. Therefore algorithms specially suited for large size problems should be used. 
Table 1. Summary of biometric databases used in the experiments

\begin{tabular}{|c|c|c|c|c|c|}
\hline Database & Biometric Traits & Matchers & Users & $\begin{array}{c}\text { Training } \\
\text { Set }\end{array}$ & $\begin{array}{c}\text { Test } \\
\text { Set }\end{array}$ \\
\hline $\begin{array}{c}\text { NIST } \\
\text { Multimodal }\end{array}$ & $\begin{array}{c}\text { Fingerprint (Left and Right) } \\
\text { Face (2 matchers) }\end{array}$ & 4 & 517 & 133645 & 133644 \\
\hline XM2VTS & $\begin{array}{c}\text { Face (5 matchers) } \\
\text { Speech (3 matchers) }\end{array}$ & 8 & 295 & 40600 & 112200 \\
\hline
\end{tabular}

\section{Experiments}

The effectiveness of the margin-based fusion rule has been evaluated on two publicly available biometric databases, namely, the partition NIST-Multimodal of the NIST-BSSR1 9] and XM2VTS Benchmark [10. The characteristics are summarized in table 1. All the data have been normalized using the min-max normalization [6]. For each experiment on NIST-Multimodal, half of the genuine and half of the impostor match scores were randomly selected to form the training set to train the fusion rules and the test set to evaluate their performance. Moreover, to avoid any bias in the following comparison, the hold out procedure has been repeated 20 times and all the reported results have been obtained as the mean values over each trial. For experiments on XM2VTS database the hold out procedure has not been implemented since we used the training-test partitioning suggested in [10].

In table 1 we also report the dimensions of training and test sets to show that in multibiometric problems the number of matchers is significantly less than the number of samples. This implies that our approach is usually involved in a linear maximization problem that faces with a low number of variables (i.e., a low-dimensional weight vector) but with a huge number of constraints (i.e., a high dimensionality of training data). In particular, on NIST-Multimodal we have only 4 matchers but 133645 samples while in XM2VTS the matchers are 8 and the number of constraints is equal to 40600. Therefore we employed the MOSEK Optimization Software [1] that provides specialized functions to solve large-scale linear programming problems.

In our first experiment we analyze the behavior of the proposed approach (hereafter called LPmax, i.e. Linear Programming maximization) on margins evaluation compared with the single matchers. To this aim, we consider the cumulative distributions of margins on the training set provided by LPmax and the single matchers. In figure 1 we report the margin cdfs for the proposed approach in comparison with the 4 matchers of the NIST-Multimodal database (figure 1,a) and the 8 matchers of XM2VTS benchmark (figure 1,b). Looking at these graphs, it could seem that some matchers exhibit higher margins. Actually, if we look at figure2 (that reports the same plots but in logarithmic scale for the $\mathrm{y}$-axis) we can note that the minimum margin of LPmax is in every case higher than all the matchers.

This agrees with the good results provided by LPmax and shown in figure 3 Here the performance on the test set have been evaluated in terms of Receiver 


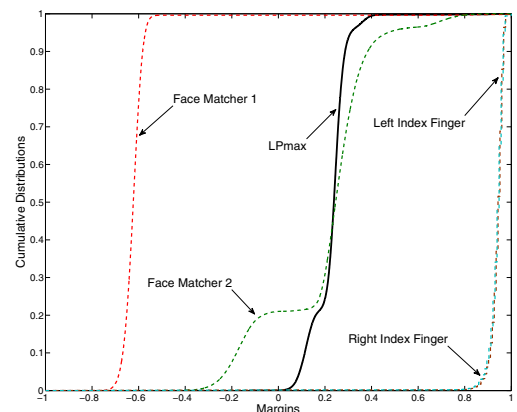

(a)

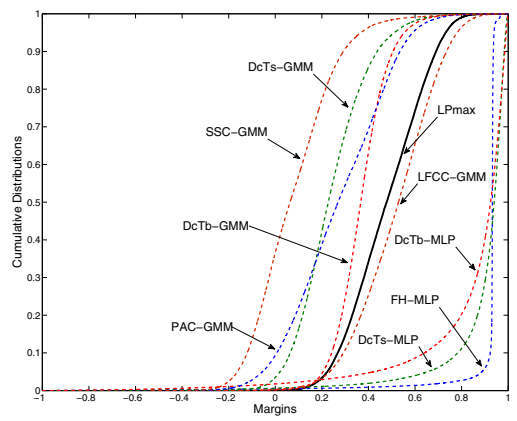

(b)

Fig. 1. Margin distributions graphs for the LPmax approach and the single matchers on the training set of the NIST-Multimodal database (a) and the XM2VTS benchmark database (b)

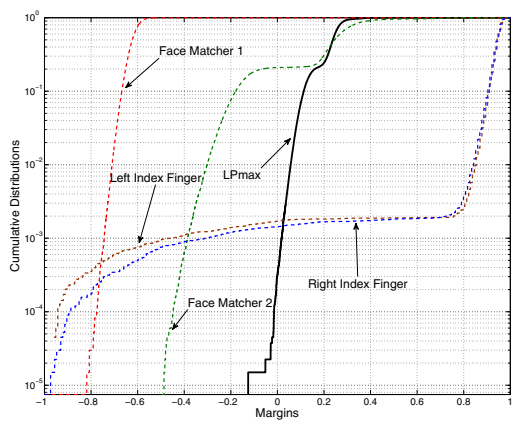

(a)

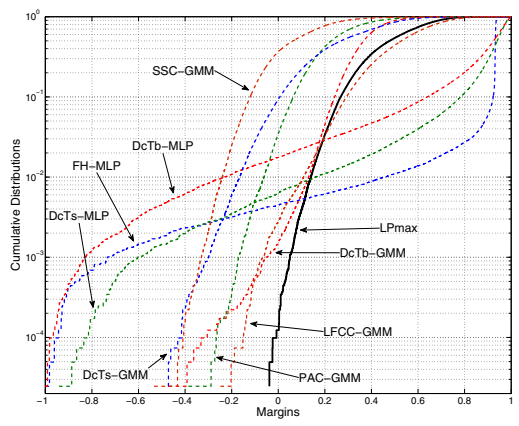

(b)

Fig. 2. The same distribution graphs of figure 1 but with logarithmic scale on y-axis

Operating Characteristic (ROC) curves that plot the Genuine Acceptance Rate (GAR) vs. the False Acceptance Rate (FAR). The graphs show the curves only with a FAR between $0.01 \%$ and $0.1 \%$ since this is the only region useful to characterize the accuracy of multibiometric systems. Graphs show that the performance of LPmax are clearly better than all the single matchers on both data sets. It is worth noting that some matchers have margin distributions sensibly more concentrated on high values than LPmax (e.g. Right Index Finger on NIST and FH-MLP on XM2VTS); however, the better performance of LPmax suggests that a high value of minimum margin is the most relevant factor for increasing the performance.

In the second experiment we have compared LPmax with other fusion techniques. In particular, we considered two classifiers as combiners, i.e., AdaBoost and Support Vector Machine (SVM) and a parametric fusion model recently proposed in 8, hereafter called GMM-LR (Gaussian Mixture Model - Likelihood 


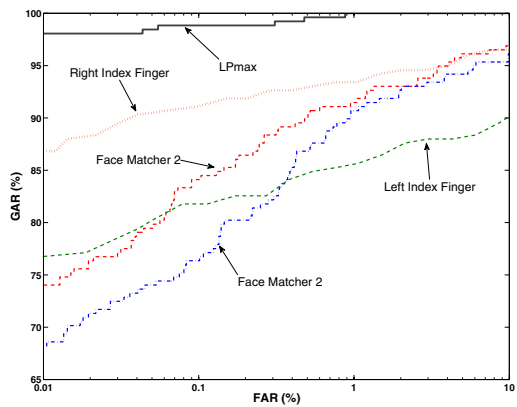

(a)

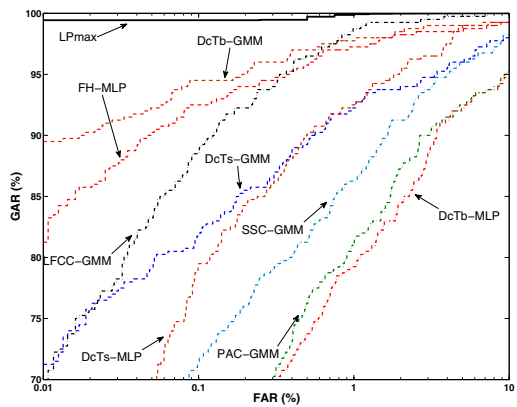

(b)

Fig. 3. Performance of LPmax compared with the matchers on the test set of the NIST-Multimodal database (a) and the XM2VTS benchmark database (b)

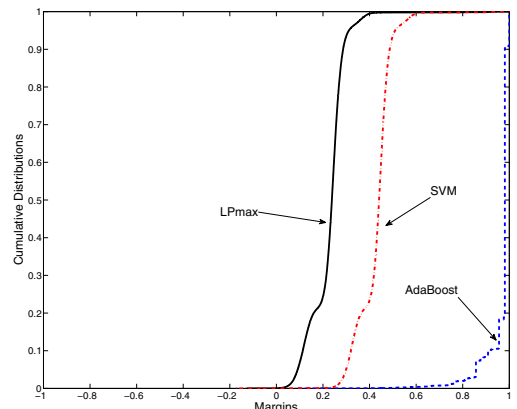

(a)

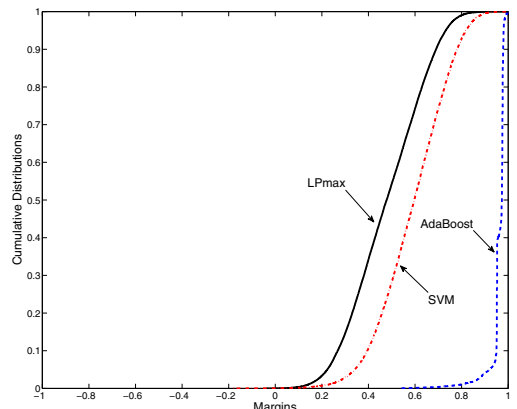

(b)

Fig. 4. Margin distributions graphs for LPmax, AdaBoost and SVM on the training set of the NIST-Multimodal database (a) and the XM2VTS benchmark database (b)

Ratio). SVM has been used since it represents a linear classifier directly built for the margin maximization while AdaBoost, even if it is a combination of linear classifiers, could be considered as the state of the art for margin-based classifiers. AdaBoost has been implemented by means of Modest Adaboost [17, a more robust and stable version of standard AdaBoost, with 300 iterations of a simple decision tree used as weak learner while SVMs have been implemented by means of SVM ${ }^{\text {light }}$ software [7. Following [8], linear SVMs have been used for both XM2VTS and NIST-Multimodal database. In all the experiments the C parameter of SVM has been put to infinite to keep fair the comparison with LPmax which does not use slack variables in the optimization algorithm.

Figure 4 shows this comparison respectively on the training set of the NISTMultimodal and XM2VTS data set:1. In this case, the performance of SVM

${ }^{1}$ GMM-LR is not based on a margin fusion approach and thus we did not consider it for margin evaluation. 


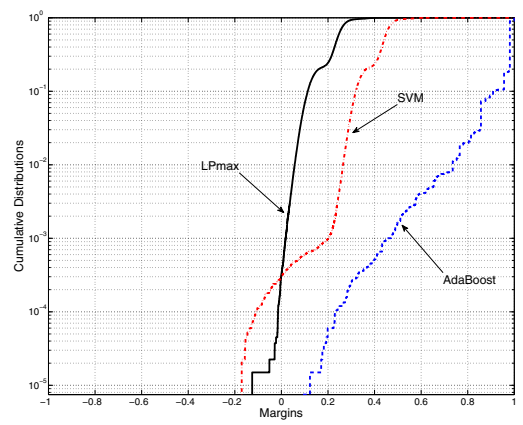

(a)

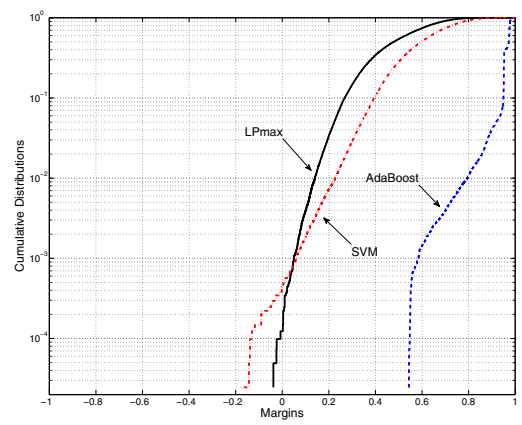

(b)

Fig. 5. The same distribution graphs of figure 4 but with logarithmic scale on y-axis

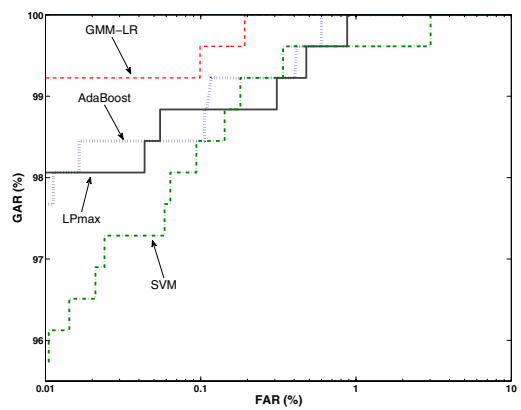

(a)

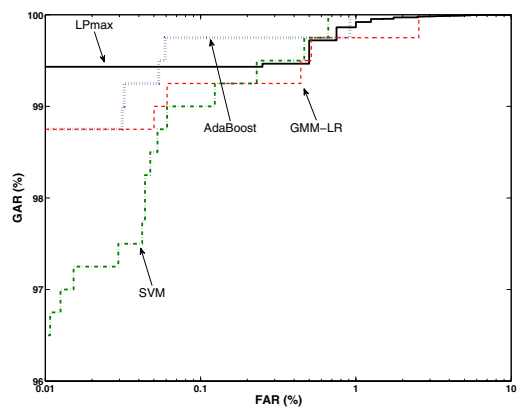

(b)

Fig. 6. Performance of the compared approaches on the test set of the NISTMultimodal database (a) and the XM2VTS benchmark database (b)

are quite good on margin distributions that have generally higher values than LPmax. However, if we look at the logarithmic graphs reported in figure 5 we can note that in both cases the minimum margin of LPmax is higher than SVM. AdaBoost, instead, exhibits clearly higher performance than the other approaches in terms of minimum margin on both data sets. As said in sect. 2 , this is due to the boosting approach that focuses on the most difficult samples of the training set to be classified, giving almost perfect results on them.

Nevertheless, if we look at the performance on the test set it is possible to note that LPmax exhibits a better generalization capability. To this aim, in figure 6 we report the ROC curves of the different approaches. Moreover, to have a single value for the performance evaluation we also report in table 2 the mean GAR obtained at a FAR of $0.01 \%$ that is the point on the y-axis of the reported ROC curves. For NIST-Multimodal we provide the mean and the standard deviation obtained from the 20 runs of the hold out procedure. 
Table 2. Performance on the test set in terms of mean GAR percentage at $0.01 \%$ FAR achieved by the different approaches. The best matchers are Right Finger and DcTb-GMM respectively for NIST-Multimodal and XM2VTS.

\begin{tabular}{|c|c|c|c|c|c|}
\hline Database & LPmax & GMM-LR & AdaBoost & SVM & Best Matcher \\
\hline NIST-Multimodal & $98.5 \pm 0.1$ & $99.1 \pm 0.4$ & $98.4 \pm 0.4$ & $95.4 \pm 1.9$ & $85.3 \pm 1.8$ \\
\hline XM2VTS & 99.5 & 98.7 & 98.8 & 96.2 & 89.5 \\
\hline
\end{tabular}

In this case, the comparison shows different results on the two data sets. On NIST-Multimodal, GMM-LR has a higher ROC curve than all the other approaches while LPmax is comparable with AdaBoost and is clearly superior to SVM and the best matcher. The better performance of GMM-LR are probably due to the score distribution of the matchers that, as shown in [8], are well fitted by the Gaussian mixture model. On XM2VTS, instead, LPmax exhibits better performance than all the other methods even if for some FAR values the ROC of AdaBoost is higher than LPmax curve. However, if we focus on table 2, we can note that, when fixing the threshold at $0.01 \%$ FAR, LPmax has higher performance on XM2VTS and only GMM-LR exhibits better performance on NIST-Multimodal even if in this case the standard deviation of LPmax is tighter than GMM-LR, thus leading to a quite small difference between the two approaches.

Some final considerations could be made about the comparison with the SVM based combination which, in some regards, provides the most similar approach to LPmax: in fact both of them are linear combination and are based on margin maximization. The only difference is that SVM provide an optimal separating hyperplane whose margins from the two classes are equal; in other words, LPmax does not have the constraint of simmetrycal margins affecting the SVM learning algorithm and this could lead to a more effective minimum margin maximization.

\section{Conclusions and Future Work}

We have illustrated a framework for the fusion of match scores in a multibiometric system based on the margin maximization. Our approach relies on the evaluation of the weights of a convex combination of matchers such that the margin of the combination on the training set is maximized. The obtained results in comparisons with other recent and commonly employed approaches both on margin distribution and on classification performance encourage a deeper analysis of the proposed method. In particular, a relaxation of the constraint in the margin maximization could be adopted by introducing slack variables to face with noisy data and a more extensive experimentation considering other biometric datasets could be employed to better verify the effectiveness of this approach. 


\section{References}

1. Mosek 2005. The MOSEK optimization tools version 5.0. User's manual and reference. MOSEK ApS, Denmark (2005)

2. Anthony, M., Bartlett, P.L.: Neural Network Learning: Theoretical Foundations. Cambridge University Press, Cambridge (1999)

3. Bartlett, P.L.: The sample complexity of pattern classification with neural networks: the size of the weights is more important than the size of the network. Information Theory, IEEE Transactions 44(2), 525-536 (1998)

4. Crammer, K., Gilad-Bachrach, R., Navot, A., Tishby, N.: Margin analysis of the lvq algorithm. In: Thrun, S., Becker, S., Obermayer, K. (eds.) Advances in Neural Information Processing Systems, vol. 15, pp. 462-469. MIT Press, Cambridge (2003)

5. Grove, A.J., Schuurmans, D.: Boosting in the limit: maximizing the margin of learned ensembles. In: AAAI 1998/IAAI 1998: Proceedings of the fifteenth national/tenth conference on Artificial intelligence/Innovative applications of artificial intelligence, Menlo Park, CA, USA, pp. 692-699. American Association for Artificial Intelligence (1998)

6. Jain, A.K., Nandakumar, K., Ross, A.: Score normalization in multimodal biometric systems. Pattern Recognition 38(12), 2270-2285 (2005)

7. Joachims, T.: Making large-scale svm learning practical. In: Schölkopf, et al. (eds.) Advances in Kernel Methods-Support Vector Learning, pp. 169-184. MIT Press, Cambridge (1999)

8. Nandakumar, K., Chen, Y., Dass, S.C., Jain, A.K.: Likelihood ratio-based biometric score fusion. Pattern Analysis and Machine Intelligence, IEEE Transactions 30(2), 342-347 (2008)

9. National Institute of Standards and Technology. Nist biometric scores set - release 1 (2004), http://www.itl.nist.gov/iad/894.03/biometricscores

10. Poh, N., Bengio, S.: Database protocols and tools for evaluating score-level fusion algorithms in biometric authentication. Pattern Recognition 39(2), 223-233 (2006)

11. Ratsch, G., Onoda, T., Muller, K.-R.: Soft margins for adaboost. Machine Learning 42(3), 287-320 (2001)

12. Schapire, R.E., Freund, Y., Bartlett, P., Lee, W.S.: Boosting the margin: A new explanation for the effectiveness of voting methods. The Annals of Statistics 26(5), 1651-1686 (1998)

13. Shawe-Taylor, J., Bartlett, P.L., Williamson, R.C., Anthony, M.: Structural risk minimization over data-dependent hierarchies. Information Theory, IEEE Transactions 44(5), 1926-1940 (1998)

14. Snelick, R., Uludag, U., Mink, A., Indovina, M., Jain, A.K.: Large-scale evaluation of multimodal biometric authentication using state-of-the-art systems. Pattern Analysis and Machine Intelligence, IEEE Transactions 27(3), 450-455 (2005)

15. Toh, K.-A., Kim, J., Lee, S.: Biometric scores fusion based on total error rate minimization. Pattern Recognition 41(3), 1066-1082 (2008)

16. Vanderbei, R.J.: Linear Programming: Foundations and Extensions, 2nd edn. Springer, Heidelberg (2001)

17. Vezhnevets, A., Vezhnevets, V.: Modest adaboost - teaching adaboost to generalize better. Graphicon-2005, Novosibirsk Akademgorodok, Russia (2005), http:// research.graphicon.ru/machine-learning/gml-adaboost-matlab-toolbox-3. html 